\section{Sektion HNO}

$D^{2}$ a die Anzahl der HNO-Kollegen, die in den Bereichen Allergologie und klinische Immunologie wissenschaftlich aktiv sind, in Deutschland noch recht klein ist, sind viele Mitglieder der Sektion HNO der Deutschen Gesellschaft für Allergologie und klinische Immunologie (DGAI) auch gleichzeitig in der Arbeitsgemeinschaft klinische Immunologie, Allergologie und Umweltmedizin der Deutschen Gesellschaft für Hals-Nasen-Ohren-Heilkunde, Kopf- und Hals-Chirurgie vertreten. Diese Personalunion entfaltet synergistische Effekte und erlaubt es, trotz der für die Bedeutung des Bereiches kleinen Gefolgschaft die Fahne der HNOHeilkunde in der Allergologie hoch zu halten und gleichzeitig auf die Rolle allergischer Erkrankungen in der HNO-Heilkunde aufmerksam zu machen.

Die Arbeit der Sektion besteht vor allem in der Erarbeitung von Themenvorschlägen für die Kongresse der DGAI und damit in der Einbindung bereits etablierter, aber vor allem auch jüngerer Kollegen in die wissenschaftliche Szene. Dies ist, so meine ich, in den letzten Jahren zunehmend gelungen. Ein weiteres Aufgabengebiet besteht in der Erarbeitung von Positionspapieren und Leitlinien, die zu einer Verbesserung der Qualität der Patientenversor-

\section{Dank an die DGAI-Sponsoren}

Die Deutsche Gesellschaft für Allergologie und klinische Immunologie dankt ihren Hauptsponsoren

ALK-Scherax Arzneimittel GmbH Allergopharma Joachim Ganzer KG Aventis Pharma Deutschland GmbH Bencard Allergie $\mathrm{GmbH}$

Essex Pharma GmbH

HAL Allergie $\mathrm{GmbH}$

Novartis Consumer Health $\mathrm{GmbH}$

Novartis Pharma GmbH

Pharmacia Diagnostics GmbH\&Co.KG

UCB Pharma GmbH gung, basierend auf aktuellen wissenschaftlichen Erkenntnissen, beitragen. Die Sektion hat dabei vor allem eine der häufigsten allergischen Erkrankungen überhaupt, die allergische Rhinitis und ihre Folgeerkrankungen, im Auge. So konnte gerade ein Positionspapier zur „Durchführung des nasalen Provokationstests bei Erkrankungen der oberen Atemwege" (S. 29-36 in dieser Ausgabe von Allergo Journal) zusammen mit der Arbeitsgemeinschaft der HNO-Gesellschaft fertiggestellt werden. „Leitlinien zur allergischen Rhinitis" sind in Vorbereitung. Hoffen wir, dass diesen Papieren die ihnen gebührende Aufmerksamkeit zu Teil wird!
Die allergische Rhinitis, lange Zeit als Banalität abgetan, steht in letzter Zeit vornehmlich als Risikofaktor für Folgeerkrankungen wie Asthma und Sinusitis im Mittelpunkt des Interesses, der so genannte „Etagenwechsel“ erhält schärfere Konturen. Dies sollte für jeden rhinologisch interessierten HNOArzt eine Aufforderung sein, sich gerade mit der Allergologie und Immunologie des Fachgebietes zu beschäftigen!

Machen Sie mit!
Prof. Dr. Claus Bachert
Sprecher der Sektion HNO
ZU Gent, Kliniek voor Neus-, Keel- en
Oorheelkunde
De Pintelaan 185, B-900o Gent/Belgien
Telefax: 0032/9/2404993
E-Mail: claus.bachert@rug.ac.be

\title{
Nachruf auf Gerhart Erdmann
}

\author{
Nur kurz nach seinem achtzigsten Geburtstag am 12. Juni 2001 \\ verstarb der Kinderarzt und Allergologe Professor Dr. med. Gerhart \\ Erdmann.
}

V or 40 Jahren hatte er als ein Pionier der deutschen Allergologie in Mainz seine Professur angetreten und dort 25 lange Jahre gearbeitet.

Seine Patienten und seine Schüler hoben immer die Warmherzigkeit und sein persönliches Engagement hervor, mit der er sich auch unter schwierigen Bedingungen um seine kleinen und großen Patienten gekümmert hat. Aufwendige Apparatemedizin und ein großes Labor standen ihm nicht zur Verfügung, trotzdem war er weithin bekannt und als Ratgeber gesucht.

Als Lehrer und erfahrener Kliniker vermittelte er seinen Schülern die Vielfalt allergologischer und pneumologischer Krankheitsbilder wie auch das Ineinandergreifen von Umwelteinflüssen und individueller Lebensführung.

Erdmann war ganzheitlich orientiert längst bevor der Begriff der Ganzheitsmedizin modern bzw. modisch wurde. Er gehörte zu den Begründern der Zeitschrift „Allergologie“, seine vie- len Mitteilungen bezeugen sein reges wissenschaftliches Interesse. Er hat wesentlich dazu beigetragen, dass die Allergologie in der Kinderheilkunde und die Kinderheilkunde in der Allergologie ernst genommen wurden. Er hat - dank der ihm eigenen Bescheidenheit - aber nie polarisiert, sondern sich um Integration bemüht. Die pädiatrische Allergologie verdankt ihm viel.

Ich persönlich freue mich darüber, ihm noch zu Lebzeiten stellvertretend den Dank seiner Schüler und Patienten übermittelt zu haben.

Er hat sich besonders über den folgenden Satz in seiner Laudatio gefreut [Allergologie 2001; 24: 272]: „Von bleibendem Wert wird sicher die Haltung sein, die Sie gemeinsam mit anderen Pionieren der Allergologie (...) eingenommen haben: das sorgfältige Interesse am einzelnen Patienten, am einzelnen Kind“.

Dies könnte ein Vermächtnis sein!

Prof. Dr. W. Dorsch, München 